\title{
Magnitude and Economic Impact of Inappropriate Use of Stress Ulcer Prophylaxis in Non-ICU Hospitalized Patients
}

Joel J. Heidelbaugh, M.D., ${ }^{1}$ and John M. Inadomi, M.D. ${ }^{2}$

${ }^{1}$ Clinical Assistant Professor, Department of Family Medicine, University of Michigan Medical School, Ann Arbor, Michigan, and Medical Director, Ypsilanti Health Center, Ypsilanti, Michigan; ${ }^{2}$ GI Outcomes and Health Services Research, University of California, San Francisco, and Division of Gastroenterology, San Francisco General Hospital, San Francisco, California

BACKGROUND: Although administration of stress ulcer prophylaxis (SUP) has been recommended for specific patients in the intensive care unit (ICU) setting, this practice has been extrapolated to care of non-ICU patients without evidence to support need or efficacy.

AIMS: $\quad$ To examine the practice of SUP in non-ICU patients in a university hospital setting, with specific attention to resource utilization.

METHODS: $\quad$ Retrospective chart review of adult non-ICU admissions to one family medicine and five general internal medicine teaching services over a consecutive 4-month period. Proportion of patients prescribed SUP was ascertained after exclusion of patients admitted on antisecretory therapy (AST) or prescribed AST for non-SUP indications. Annual cost estimates were calculated assuming full compliance.

RESULTS: $\quad$ Of 1,769 patient admissions, $22 \%$ received SUP and $54 \%$ of these were discharged home on AST. None of these patients met evidence-based criteria for appropriate SUP. Inpatient SUP cost $\$ 11,024$ over the 4 months of the study (\$44,096 annually), and outpatient costs based on discharge prescriptions were $\$ 16,924$ ( $\$ 67,695$ annually), yielding a total cost expenditure of $\$ 27,948$ (\$111,791 annually).

CONCLUSION: SUP is overutilized in the non-ICU setting, and patients are often discharged unnecessarily on AST, resulting in significant cost expenditure. Interventions to ensure appropriate use of SUP should decrease resource expenditures without detrimental impact on quality of care.

(Am J Gastroenterol 2006;101:2200-2205)

\section{INTRODUCTION}

Stress ulceration is defined as a form of hemorrhagic gastritis that may occur in patients who have suffered a moderate to severe physiologically stressful event such as surgery, trauma, organ failure, sepsis, thermal injury, or prolonged mechanical ventilation (1). Since not all episodes of microscopic bleeding are clinically relevant, studies using only microscopic bleeding as an end point artificially inflate the reported frequency of gastrointestinal (GI) bleeding and stress ulceration (2). Previous studies that have provided estimates of stress ulceration incidence in the intensive care unit (ICU) setting may have led providers to consider this potential problem in patients in non-ICU settings, leading to significant patient care and cost implications.

The American Society of Health-System Pharmacists (ASHP) guidelines for stress ulcer prophylaxis (SUP) from 1999 serve as a framework for instituting preventive therapy in ICU patients. SUP is not recommended for adult general medical and surgical patients in non-ICU settings with fewer than two risk factors for clinically important bleeding, or for patients with two or more risk factors (Table 1). Few studies to date have effectively examined the role of SUP in non-ICU patients (3-5). Cost-effectiveness studies in patients receiving SUP have been limited solely to ICU settings (6-9).

Our hypothesis is that many patients admitted to general medical and family medicine (non-ICU) services are routinely placed on antisecretory therapy (AST) for SUP, when neither the admitting nor the comorbid diagnoses support their use for either treatment or GI prophylaxis. Moreover, we suspect that a large percentage of these patients started on AST for SUP are discharged from the hospital on these medications. Our goal was to examine the practice of SUP in non-ICU patients in a university hospital setting, with specific attention to resource utilization.

\section{METHODS}

The University of Michigan Health System is a large, multispecialty teaching hospital located in Ann Arbor, Michigan. 


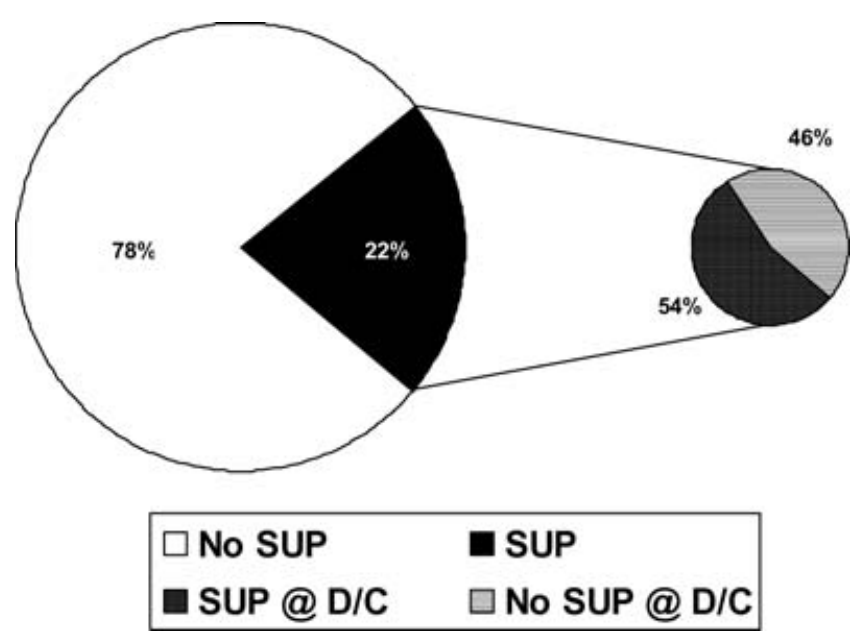

Figure 1. Prevalence of stress ulcer prophylaxis in non-ICU patients. Proportion of consecutive patient admissions over 4-month period of study in which stress ulcer prophylaxis (SUP) was documented to have been administered. The subset on the right depicts the proportion administered SUP while as inpatients who were subsequently discharged on antisecretory therapy. @ D/C = at time of discharge; ICU $=$ intensive care unit.

orthopedic (2.1), pulmonary (1.8), renal (1.8), and rheumatologic (2.2) (Table 3).

Inpatient SUP costs for the study cohort totaled $\$ 11,024$ over the 4 months of the study. Assuming constant rates of SUP prescription, this represents an annual outlay of $\$ 44,096$ for the six admitting services. Outpatient pharmacy costs totaled $\$ 16,924$ on the basis of the prescriptions given at discharge. Assuming full compliance by patients and a constant rate of prescription, this results in an annual cost of $\$ 67,695$. Total AST costs for the study cohort, combining inpatient and outpatient medications, equaled $\$ 27,948$, which represents an

Table 2. Incidence of SUP by Diagnosis

\begin{tabular}{lccc}
\hline Organ System & $\begin{array}{c}\text { Number of } \\
\text { Admissions }\end{array}$ & $\begin{array}{c}\% \text { of } \\
\text { Total }\end{array}$ & $\begin{array}{c}\% \text { That } \\
\text { Received SUP }\end{array}$ \\
\hline Gastrointestinal & 360 & $20.3 \%$ & $15.6 \%$ \\
Infectious diseases & 250 & $14.1 \%$ & $22.4 \%$ \\
Pulmonary & 242 & $13.7 \%$ & $27.7 \%$ \\
Cardiovascular & 196 & $11.1 \%$ & $30.1 \%$ \\
Metabolic & 179 & $10.1 \%$ & $21.2 \%$ \\
Renal & 142 & $8.0 \%$ & $30.3 \%$ \\
Neurological & 127 & $7.2 \%$ & $20.5 \%$ \\
Urologic & 115 & $6.5 \%$ & $18.3 \%$ \\
Orthopedic & 98 & $5.5 \%$ & $29.6 \%$ \\
Otherł & 87 & $4.9 \%$ & $10.3 \%$ \\
Hematologic & 72 & $4.1 \%$ & $27.8 \%$ \\
Rheumatologic & 53 & $3.0 \%$ & $32.1 \%$ \\
Psychologic & 30 & $1.7 \%$ & $16.7 \%$ \\
\hline
\end{tabular}

${ }^{*}$ Refers to primary diagnosis listed on admission and discharge records for each patient admission. Note that for some patients, multiple primary diagnoses were listed. ${ }^{\dagger}$ Reflects patient admissions treated with antisecretory medications as specifically documented for SUP, not for treatment of disease.

$\ddagger$ Reflects admission and discharge diagnoses listed under the categories: dental, dermatologic, drug reaction, gynecologic, obstetrical, oncologic, ophthalmologic, otolaryngologic, and trauma.
Table 3. SUP by Admission Diagnosis

\begin{tabular}{lcc}
\hline & Odds Ratio & 95\% Confidence Interval \\
\hline Cardiology & 2.0 & $1.4-2.9$ \\
Endocrinology & 0.3 & $.04-2.3$ \\
Hematology & 1.8 & $1.1-3.2$ \\
Metabolism & 1.3 & $0.9-2.0$ \\
Infectious disease & 1.4 & $0.9-2.0$ \\
Neurology & 1.2 & $0.8-2.0$ \\
Orthopedic & 2.1 & $1.3-3.5$ \\
Pulmonary & 1.8 & $1.2-2.5$ \\
Psychiatry & 1.0 & $0.4-2.7$ \\
Renal & 1.8 & $1.2-2.7$ \\
Rheumatology & 2.2 & $1.2-4.1$ \\
Urology & 1.1 & $0.6-1.8$ \\
Other & 0.6 & $0.3-1.2$ \\
\hline
\end{tabular}

SUP $=$ stress ulcer prophylaxis.

Other-dental, dermatologic, drug reaction, gynecologic, obstetrical, oncologic, ophthalmologic, otolaryngologic, and trauma.

annual total cost for AST prescribed for unnecessary SUP of $\$ 111,791$ (Fig. 2).

\section{DISCUSSION}

Despite a lack of evidence supporting its use, this study revealed that $22.1 \%$ of hospitalized non-ICU patients were routinely prescribed SUP. More disturbingly, the majority of these patients (54.4\%) unnecessarily continued AST in the outpatient setting. This practice resulted in substantial cost expenditure that could be avoided. While guidelines for SUP in ICU patients have been well defined in the

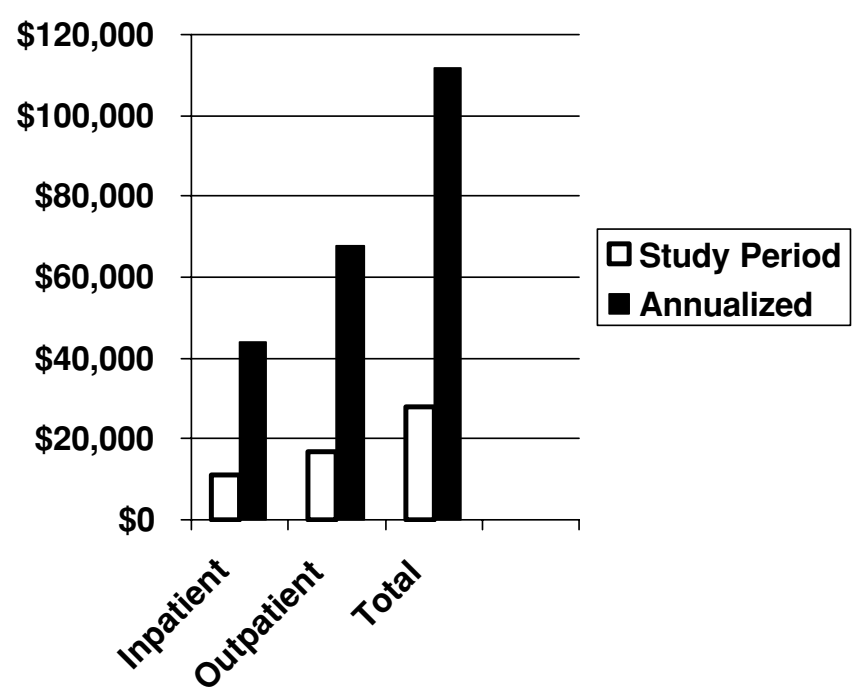

Figure 2. Cost of stress ulcer prophylaxis in non-ICU patients. Cost of stress ulcer prophylaxis (SUP) based on pharmacy expenditures for the six admitting services examined in this report. Data are separated by costs incurred as inpatient (based on inpatient pharmacy records) and outpatient (based on discharge medication prescriptions), as well as by total costs. Annualized data assume that a constant proportion of patients are prescribed SUP, and that patients are fully compliant with medication. ICU = intensive care unit. 
medical literature, the perceived benefit from SUP has been extrapolated to patients in non-ICU settings (assuming similar criteria and similar appropriate guidelines), leading to an excessive consumption of these medications and increased overall cost.

Our study highlights the common practice of nonindicated SUP use, as well as the high rate of AST without documented indication among patients admitted to non-ICU wards. There was no difference among admitting specialties; therefore, this likely represents a systematic practice not limited to one service. Interestingly, patients admitted with a primary GI diagnosis were less likely to receive SUP than several other diagnostic categories, notably patients admitted with rheumatologic disorders, who were 2.2 times more likely to receive SUP. Although this may represent a higher use of potentially ulcerogenic medication among patients with non-GI diagnoses, the medical record documentation stated use of AST for SUP as opposed to chemoprevention against GI toxicity due to concomitant medication use.

One reason for the widespread use of SUP may be the low rate of side effects associated with these medications. Most adverse effects attributable to antacids, $\mathrm{H}_{2} \mathrm{RAs}$, and sucralfate are uncommon and occur in less than $1 \%$ of adult patients, particularly when given for a period of less than $2 \mathrm{wk}$ (2). Rare adverse side effects include aluminum and magnesium toxicity, vitamin $\mathrm{B}_{12}$ deficiency, and nosocomial pneumonia. However, it has been noted that $\mathrm{H}_{2} \mathrm{RAs}$ and PPIs have the potential for drug-drug, drug-nutrient, and drug-test interactions through a variety of mechanisms (2). The frequency and severity of adverse effects may increase in renal failure, in the elderly, and in malnourished patients, secondary to electrolyte accumulation due to antacid administration or central nervous system disturbances due to administration of $\mathrm{H}_{2}$ RAs. Reduction of gastric acid secretion by AST may increase the risk of community-acquired pneumonia. A large case-controlled study found that patients taking $\mathrm{H}_{2} \mathrm{RAs}$ had a 1.63 -fold increased risk of developing pneumonia $(95 \%$ CI) compared with those who stopped AST (10). For patients taking PPIs, a significant positive dose-response relationship was observed, while for current $\mathrm{H}_{2} \mathrm{RA}$ users, the variation in dose was restricted. Conversely, a randomized, yet much smaller study determined that the rate of the development of nosocomial pneumonia in 158 mechanically ventilated patients in a surgical ICU was not adversely affected by the administration of $\mathrm{H}_{2}$ RAs for SUP (11).

Controversy exists over the use of pharmacologically induced acid suppression in critically ill patients at risk for stress ulcers. The ASHP guideline does not include recommendations on the use of PPIs for SUP, yet we suspect they may be the most frequently used acid-suppressive medications for SUP. This guideline recommends that the choice among antisecretory agents for SUP in adult patients admitted to general medical and surgical ICUs should be made on an institution-specific basis. Expert opinion states that this choice should take into account concerns regarding administration, adverse-effect profile, and total costs (2). One review summarized that PPIs are both safe and efficacious for elevating intragastric $\mathrm{pH}$ in critically ill patients, and should be used only as an alternative to $\mathrm{H}_{2} \mathrm{RAs}$ or sucralfate, since the superiority of PPIs over these agents for preventing stress ulcers has not been established (12). Additional comparative studies with adequate patient numbers and pharmacoeconomic analyses are needed before PPIs are considered the agents of choice for SUP in either ICU or non-ICU settings (13).

On the basis of the potential adverse events, SUP prophylaxis is not warranted in patients at low risk for clinically important bleeding (e.g., patients not receiving mechanical ventilation, or those without significant coagulopathy); the number needed to treat (NNT) is greater than 900 such patients to prevent a single episode of clinically significant GI bleeding (14). ASHP guidelines highlight a study of 100 adults on a general medical and surgical hospital ward in Barcelona, Spain. Their results revealed that the frequency of all types of clinically important GI bleeding was significantly lower with prophylaxis using antacids, but only if two or more risk factors for bleeding were present prior to the initiation of SUP (15). One study of 293 patients with chronic renal failure on dialysis revealed a $41 \%$ utilization rate of AST for SUP, and in $63 \%$ of cases, there was no adequate indication (16).

Two studies published since the release of the ASHP guidelines reported significant overuse of SUP in non-ICU patients. Nardino et al. reviewed 226 patients admitted to a general adult medicine service in a community hospital and found that $54 \%$ of patients received AST, most commonly $\mathrm{H}_{2} \mathrm{RAs}$ ( $62 \%$ of total), yet $65 \%$ of prescriptions were not indicated as determined by consensus review. Among the $54 \%$ of patients who received SUP, 55\% were discharged home on the therapy (4). Similar to our findings, Zink et al. reviewed 814 general adult medicine admissions in a community hospital setting, of which 324 were given SUP ( $40 \%$ of total). They noted that $40 \%$ of patients receiving SUP were actually given AST for an appropriate medical indication (thus not SUP), while $60 \%$ were not. The most frequently cited reason for prescribing AST without an indication was "GI prophylaxis," yet a major limitation of this study was that acceptable indications for acid suppression were not recorded. Thirty-four percent of patients in that study who received SUP were discharged home on the acid-suppressive medication on which they were started (5). Resource utilization was not reported in these studies.

Resource utilization data regarding SUP trends in the ICU setting have been published. A study performed at the Carolinas Medical Center found an estimated annual savings of $\$ 102,895$ in patient charges and $\$ 11,333$ in actual drug costs in a trauma ICU attributable to the implementation of SUP guidelines (6). Another ICU-based study performed in a tertiary-care teaching hospital in Vancouver, British Columbia, Canada concluded that after the introduction of a guideline for SUP administration, appropriateness of therapy increased from $75.8 \%$ to $91.1 \%$, and medication costs decreased from C $\$ 2.50 /$ day to $\mathrm{C} \$ 1.30$ /day without any statistically significant difference in clinical outcomes. Introduction 
of this guideline was associated with an increase in appropriateness of prophylaxis and a decrease in medication costs (7).

Since the data in our study were abstracted by chart review from each patient hospitalization, it is possible that GI symptoms may not have been reported and/or documented in the patient chart. Ultimately, we suspect that the reported incidence data for SUP in our study may be artificially low, which is commensurate with the Nardino et al. and Zink et al. studies. The cost analysis was limited to the actual amount of medication dispensed by the pharmacy during the hospitalization, and the prescriptions written for the patient at the time of discharge. Extrapolation to estimate annual cost expenditures for the cohort was based on assumptions that a constant proportion of patients were administered SUP as inpatients and discharged on AST. One potentially confounding factor in our cost analysis was that we assumed full compliance with obtaining prescriptions for AST on discharge from the hospital. In retrospect, to potentially be more accurate, we could have followed each of these patient admissions to determine if they in fact filled the prescriptions for AST and if they continued on AST unnecessarily over time. Therefore, annualized cost estimates may deviate from actual costs if the 4-month period of study was not representative of usual practice, or if patients did not adhere to prescribed medication as outpatients. All admitting services had numerous attending and resident physicians rotating coverage throughout the 4 months of the study, and there were also residents who covered more than one service. Thus, this appears to be a systematic issue not limited to a particular group of residents or faculty.

Our impression is that the practice of SUP in the nonICU setting may be somewhat reflexive, extrapolated from common practice in the ICU setting. This hypothesis stems from an idea that physicians may feel that certain non-ICU patients are at a higher risk of developing stress ulcers (i.e., those patients on chronic or high-dose steroids, patients who are septic or potentially septic, etc.), and that a relatively simple intervention of using AST would provide minimal patient risk and safeguard against stress ulceration, without clear regard for cost-effective provisions of care. No data currently exist on stress ulceration rates in non-ICU patients; data from ICU populations are widely variable and not applicable to this study. We feel that it is reasonable, however, for clinician judgment to determine if a patient with moderate to severe physiologic stress (i.e., the use of chronic or high-dose steroids, sepsis, or potential sepsis, etc.) in the nonICU setting may ultimately benefit from AST for SUP, taking into consideration potential risks versus benefits, likelihood of stress ulcer development, cost-effectiveness, and certainly a plan for ensuring that patients are not discharged on AST without appropriate symptoms or indication for treatment.

Potential interventions to minimize inappropriate use of SUP in the non-ICU setting include (i) the use of the ASHP guidelines (albeit from 1999) in non-ICU patients (perhaps there will eventually be updated guidelines with solid [or even expert opinion] recommendations but, to date, no such update is currently under way), (ii) adaptation of these or very similar guidelines for institutions that are readily accessible and could be easily referenced, and (iii) appropriate instruction of resident and junior attending physicians in this practice as a model for cost-effectiveness of care. Overuse of resources is compounded by the frequent practice of continuing nonindicated AST after discharge. Provider education regarding proper implementation of SUP may lead to substantial reductions in health-care costs without impairment of patient outcome. As there are wide variations in prescribing practices for both non-ICU and ICU SUP, we reiterate previous recommendations that institutions consult published literature and use preexisting guidelines as templates for the development of their own institutional guidelines $(2,17)$. Future updated ASHP guidelines on SUP may address both the role of PPIs and resource utilization.

\section{STUDY HIGHLIGHTS}

\section{What Is Current Knowledge}

- Stress ulcer prophylaxis may be indicated in certain ICU patients.

\section{What Is New Here}

- Stress ulcer prophylaxis is overutilized in the non-ICU setting.

- There is a lack of solid evidence to support stress ulcer prophylaxis in the non-ICU setting.

- Patients are often discharged unnecessarily on antisecretory therapy.

- Ensuring appropriate use of stress ulcer prophylaxis should decrease resource expenditures.

Reprint requests and correspondence: Joel J. Heidelbaugh, M.D., Ypsilanti Health Center, 200 Arnet Suite 200, Ypsilanti, MI 48198.

Received February 14, 2006; accepted April 18, 2006.

\section{REFERENCES}

1. Anderberg B, Sjodahl R. Prophylaxis and management of stress ulcers. Scand J Gastroenterol 1985;20(suppl 110):101-4.

2. American Society of Health-System Pharmacists. ASHP therapeutic guidelines on stress ulcer prophylaxis. Am J Health-Syst Pharm 1999;56:347-79.

3. Parente F, Cucino C, Gallus S, et al. Hospital use of acidsuppressive medications and its fall-out on prescribing in general practice: A 1-month survey. Aliment Pharmacol Ther 2003;17:1503-6.

4. Nardino RJ, Vender RJ, Herbert PN. Overuse of acidsuppressive therapy in hospitalized patients. Am J Gastroenterol 2000;95:3118-22.

5. Zink DA, Pohlman M, Barnes M, et al. Long-term use of acid suppression started inappropriately during hospitalization. Aliment Pharmacol Ther 2005;21:1203-9. 
6. Mostafa G, Sing R, Matthews B, et al. The economic benefit of practice guidelines for stress ulcer prophylaxis. Am Surg 2002;68:146-50.

7. Pitimana-aree $S$, Forrest D, Brown G, et al. Implementation of a clinical practice guideline for stress ulcer prophylaxis increases appropriateness and decreases cost of care. Intensive Care Med 1998;24:217-23.

8. Devlin JW, Claire KS, Dulchavsky SA, et al. Impact of stress ulcer prophylaxis guidelines on drug cost and frequency of major gastrointestinal bleeding. Pharmacotherapy 1999; 19:452-60.

9. Schupp KN, Schrand LM, Mutnick AH. A costeffectiveness analysis of stress ulcer prophylaxis. Ann Pharmacother 2003;37:631-5.

10. Laheij RJ, Sturkenboom MC, Hassing RJ, et al. Risk of community-acquired pneumonia and use of gastric-acid suppressive drugs. JAMA 2004;292:1955-60.

11. Hanisch EW, Encke A, Naujoks F, et al. A randomized, double-blind trial for stress ulcer prophylaxis shows no evidence of increased pneumonia. Am J Surg 1998;176:453-7.

12. Sung JJ. The role of acid suppression in the management and prevention of gastrointestinal hemorrhage associated with gastroduodenal ulcers. Gastroenterol Clin North Am 2003;32(suppl 3):S11-23.

13. Jung R, MacLaren R. Proton-pump inhibitors for stress ulcer prophylaxis in critically ill patients. Ann Pharmacother 2002;36:1929-37.
14. Cash BD. Evidence-based medicine as it applies to acid suppression in the hospitalized patient. Crit Care Med 2002;30(suppl 6):S373-8.

15. Estruch R, Pedrol E, Castells A, et al. Prophylaxis of gastrointestinal tract bleeding with magaldrate in patients admitted to a general hospital ward. Scand J Gastroenterol 1991;26:819-26.

16. Strid H, Simren M, Bjornsson ES. Overuse of acid suppressant drugs in patients with chronic renal failure. Nephrol Dial Transplant 2003;18:570-5.

17. Erstad BL, Jeffrey F, Barletta JF, et al. Survey of stress ulcer prophylaxis. Crit Care 1999;3:145-9.

\section{CONFLICT OF INTEREST}

Guarantor of the article: Joel Heidelbaugh, M.D. and John Inadomi, M.D.

Specific author contributions: John Inadomi, M.D.: study design, statistical analysis, approval of final paper Joel Heidelbaugh, M.D.: study design, statistical analysis, approval of final paper, and composition of initial draft and procurement of study data.

Financial support: No financial conflicts of interest. 